\title{
Long-term Variability in Be-Star disks
}

\author{
J.H. Telting \\ Isaac Newton Group of Telescopes, Netherlands Organization for \\ Scientific Research, Apartado 321, Santa Cruz de La Palma, Spain
}

\begin{abstract}
.
Be stars can switch between non-disk, gradual disk growth, disk-loss events, and shell-line phases. Many aspects of this Be phenomenon are still not understood. In this paper I review recent work on variability in Be-star disks, divided in four different topics: disk growth (Section 1), long-term $V / R$ variations and global disk oscillations (Section 2), spectacular variations (Section 3), and, concisely, the disk variability in Be/X-ray binaries (Section 4).
\end{abstract}

\section{Disk growth}

In current Be-star research a lot of emphasis is put on the question of what stellar mass-loss mechanism can feed the equatorial disks around these stars (see e.g. Baade in these proc.) Disks may be fed through atmospheric outbursts of magnetic or pulsational origin, through rotational compression of the 'normal' high-speed stellar wind, through mass-loss induced by (near-)critical rotation, or by other mechanisms that have been proposed. An observational test of these proposed mass-loss mechanisms is provided by the observed growth of disks around Be stars. Disk growth is best determined for stars that go through cycles of disk-loss, nondisk, and subsequent disk-growth stages. Here I have collected some observational material trying to establish the disk-growth rates.

Recent work presenting or discussing observations of disk variability, disk development or disk growth, is listed by object: $\gamma$ Cas (Smith et al. 1998, Smith \& Robinson 1999); $\epsilon$ Per (Gies et al. in press); $\omega$ CMa (Balona et al. 1999, Harmanec 1998, Stefl et al. 1999); $\mu$ Cen (Hanuschik et al. 1993, Peters 1998, Rivinius et al. 1998); HD76534 (Oudmaijer \& Drew 1997); $\lambda$ Eri (Mennickent et al. 1998, Smith et al. 1994/1996/1997ab ); 48 Lib (Hanuschik \& Vrancken 1996); $\kappa$ Dra (Juza et al. 1994, Hirata 1995); V744 Her (Hirata 1995); BU Tau (Pleione, Hirata 1995); 4 Her (Koubsky et al. 1997).

Similar papers on disk variability in Be/X-ray binaries are: X Per (Kunjaya \& Hirata 1995, Roche et al. 1997, Tarasov \& Roche 1995, Telting et al. 1998); V725 Tau/A0535+26 (Clark et al. 1998ab/1999, Hao et al. 1996); 4U0728-25 (Negueruela et al. 1996); LSI+61235/RX J0146.9+612 (Reig et al. 1997a); EXO 2030+375 (Reig et al. 1998); V635 Cas/4U0115+63 (Negueruela et al. 1997, Unger et al. 1998).

Very useful and revealing survey work has been presented by Hubert \& Floquet (1998), Moujtahid et al. (1998), and Pavlovski et al. (1997). 
Using IRAS observations Waters et al. $(1986,1987)$ showed for a large sample of Be stars that the infrared flux excesses of Be stars can be understood by free-free and free-bound emission arising from the circumstellar equatorial disk (see also Gehrz et al. 1974, Kastner \& Mazzali 1989). Using a simple geometry of a disk with constant opening angle and a power law radial density distribution Waters et al. derived disk densities and estimated the mass-loss rates in the disk.

From vast observational effort to measure variability of Be-star disks, and in particular the growth of disks (Rivinius et al. 1998, Roche et al. 1997), diskgrowth rates have now been measured for three stars: X Per (Telting et al. 1998), $\mu$ Cen (Hanuschik et al. 1993) and Pleione (= BU Tau; Hirata 1995). The data for $\mu$ Cen show that the stellar mass-loss mechanism that is responsible for the growth of its disk involves photospheric outbursts which may be triggered by superposition of different non-radial pulsation modes (Rivinius et al. 1998). It is not yet clear whether this mechanism is the main contributor to the growth of the disks around all Be stars of all different spectral subtypes.

In this paper I compare the disk-growth rates, as determined from variability studies uncovering the growth of a disk after a diskless phase, with the statically derived (from IRAS snap shots) values of mass-loss rates in the disk. In essence the two methods measure the same quantity; one can use the data of the three stars mentioned above to calibrate the IRAS results.

The mass-loss rates and disk densities show dependence on the spectral subtype: the earlier the spectral type, the denser is the disk and the higher is the mass-loss rate or disk-growth rate. Whichever model one accepts as the stellar mass-loss mechanism that feeds the disks around Be stars, it should account for this observed spectral dependence of disk density and growth rate.

\subsection{Observed disk-growth rates}

The disk-growth rate has been reported for three Be stars in the literature. In each of these cases the star had undergone a diskless phase, after which the build up of a new disk could be monitored. From determining the mass content of the disk at different epochs, various authors (see below) could estimate the growth rate of these disks. Note that for each of these stars the disk-growth rate has been determined independently using different methods. It is probably fair to estimate the error on these derived growth rates as at least a factor of two.

$X$ Per X Per (HD24534) is a B0Ve star (Lyubimkov et al. 1996, Roche et al. 1997 ) and is the optical component of the high mass X-ray binary $4 \mathrm{U} 0352+30$. Smith \& Roche (1999) estimate the orbital period as 20-30 years. With $v \sin i$ $\sim 150-200 \mathrm{~km} / \mathrm{s}$ (Slettebak 1982, Reynolds et al. 1992), the star is thought to be seen at a low or intermediate inclination angle.

During the last decades X Per underwent a series of disk-loss and build-up events (e.g. Roche et al. 1993). Using optical and near infrared photometry Telting et al. (1998) modelled the free-free and free-bound emission from the disk as a function of time (they used an adaptation of the model introduced by Waters et al. 1986). Closely following the disk build up of 1993-1995 they could derive the mass content of the disk as a function of time, and they determined a disk growth rate of about $4.5 \times 10^{-9} \mathrm{M}_{\odot} /$ year . 
$\mu$ Cen The B2IV-Ve star $\mu$ Cen (HD120324) has a relatively low projected rotational velocity $(v \sin i \sim 130 \mathrm{~km} / \mathrm{s}$, Brown \& Verschueren 1997) and is probably seen under a moderate inclination angle. The star is known to show photospheric outbursts (e.g. Hanuschik et al. 1993, Rivinius et al. 1998), which lead to the expulsion of matter from the star. The outbursts are followed by a narrowing of the peak separation of the double peaked $\mathrm{Fe}$ and $\mathrm{H}$ emission lines, which is clear evidence for the outflow of the expelled matter. Rivinius et al. (1998b) have shown that the outbursts appear when the non-radial pulsation modes of the star superimpose constructively.

$\mu$ Cen was observed to be in a diskless phase between 1977 and 1989 (see e.g. Rivinius et al. 1998 and references therein), after which the disk began building up again. Hanuschik et al. (1993) have monitored the $\mathrm{H} \alpha$ line strength during a. few of the outbursts and derived a disk-growth rate of about $4 \times 10^{-9} \mathrm{M}_{\odot} /$ year during the outbursts. This finding combined with the burst rates reported by Rivinius et al. (1998) and Hubert \& Floquet (1998 from Hipparcos data) leads to an estimate of the disk-growth rate of this star of $2 \times 10^{-9} \mathrm{M}_{\odot} /$ year.

Pleione Pleione (BU Tau, HD23862) is a B8Ve star that can show long-lived shell lines and is known to switch between $\mathrm{B}, \mathrm{Be}$ and $\mathrm{B}$-shell phases. As the projected rotational velocity is a considerable fraction of the critical velocity $(v \sin i \sim 320 \mathrm{~km} / \mathrm{s}$, Slettebak 1982) the star must be seen nearly equator on.

Hirata (1995) presented the observational history of this star for the last decades, showing the gradual increase of absorption strength of the Fe II shell lines from 1974-1981 during a phase of disk growth. The disk growth was preceded by a phase that lasted two years. During this time the star moved through the visual colour-magnitude diagram consistent with the trajectory expected for an equator-on star that is gradually spinning up to the critical rotation velocity. From the gradual increase of the absorption strength of the Fe II shell lines, Hirata derived the disk-growth rate of this star of about $6 \times 10^{-11} \mathrm{M}_{\odot} /$ year .

\subsection{Modelling the IR flux excess}

The disk-growth rates of the three Be stars are plotted as a function of spectral subtype in the bottom panel of Figure 1. The solid straight line is a least-squares fit to these points.

As argued above, the observed disk-growth rates should be compatible with the disk mass-loss rates as derived from the IR flux excesses as measured by the IRAS satellite. In the bottom panel of Figure 1 the disk mass-loss rates as derived by Waters et al. (1987) are plotted, where I took for each spectral subtype the average of the values given for all the luminosity class $\mathrm{V}$ stars in their sample. A total of 27 stars contribute to these data. These data are least-squares fitted with a straight line.

From the bottom panel of Figure 1, it becomes clear immediately that the two datasets have the same spectral type dependence, but that the mass-loss rates as estimated from the IR snap shot excesses are about twelve times higher than the dynamically determined disk-growth rates. The fact that the slope of the fits to both datasets is the same validates the aim to calibrate the IR disk mass-loss rates using the observed disk-growth rates. 

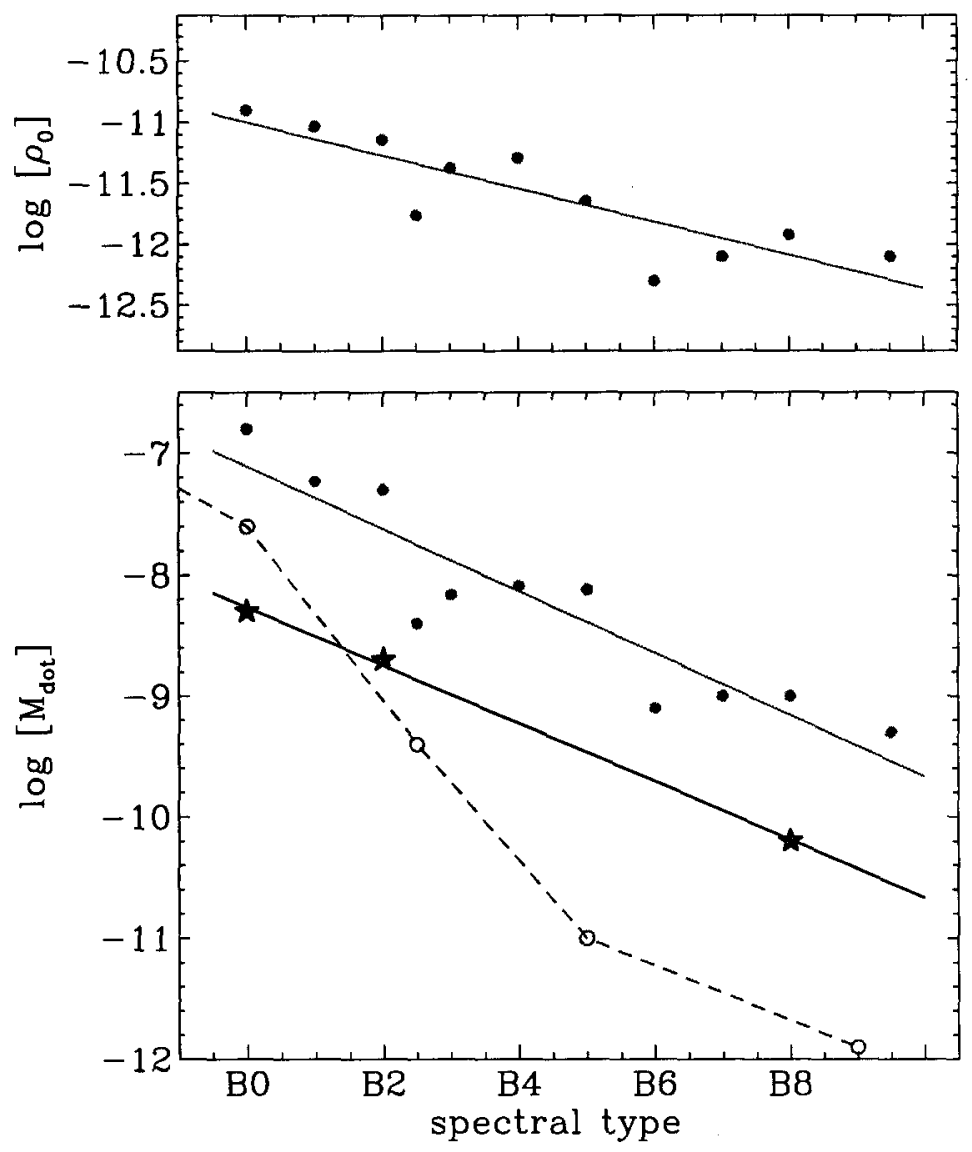

Figure 1. Top: disk-base density $\rho_{0}$, as measured from IR continuum excesses of stars in the IRAS sample (Waters et al. 1987), as a function of spectral type.

Bottom: Star symbols with solid line: dynamically measured diskgrowth rates of X Per, $\mu$ Cen and Pleione (see text) and least squares fit. Filled circles with solid line : disk mass-loss rates derived from IRAS disk densities (Waters et al. 1987) and least squares fit. Open circles with dashed line: theoretical mass-loss rates for ordinary B stars (Kudritzki et al. 1989) as tabulated by Bjorkman \& Cassinelli (1993).

In order to estimate the IR disk mass-loss rates Waters et al. (1987) had to make assumptions regarding the disk opening angle, $\theta$, and outflow speed at the base of the disk, $v_{0}$, the latter being linked to the radial density distribution through the equation of mass continuity. Below I argue that the difference of the factor of twelve in Figure 1 can easily be accounted for if one allows more modern values for these parameters. 
Disk densities derived from IRAS data Using a simple disk model Waters et al. (1987) modelled the disk-base densities of the Be stars in the IRAS sample. Assuming that the IR continuum excesses are mainly due to free-free and free-bound emission in the disk, they derived typical disk-base densities $10^{-12} \leqslant \rho_{0} \lesssim 10^{-11} \mathrm{gram} / \mathrm{cm}^{3}$ (see also e.g. Gehrz et al. 1974, Poeckert \& Marlborough 1978, Kastner \& Mazzali 1989, Johnston et al. 1996). In the top panel of Figure 1 these densities are plotted as a function of spectral type. For each of the subtypes I averaged the derived densities for all measured luminosity class V stars. A total of 28 stars contribute to the plot; a least squares fit to the data is drawn as well. One can clearly see that the disk-base densities of early-type Be stars are denser than those of late-type Be-stars: the density difference is about a factor of twenty.

Note that new modelling results (see Millar, these proc.) show that disk temperatures may be considerably lower than assumed by the above authors, which may have an impact on the derived disk-base densities, but not so much on the dependence of disk-base densities and mass-loss rates on spectral type.

Mass loss rates derived from IRAS data Assuming gradual outflow in the disk, Waters et al. (1987) could estimate the mass-loss rates in the disk from

$$
\dot{M}=C \cdot v_{0} \cdot \sin (\theta) \cdot \rho_{0} \cdot R_{*}^{2}
$$

with $v_{0}$ and $\theta$ chosen as $5 \mathrm{~km} / \mathrm{s}$ and $15^{\circ}$ respectively. It is clear from this equation that the spectral type dependence of $\dot{M}$ comes in through the base density and stellar radius. Once again I stress that this observed slope is the same as that observed from the dynamically determined disk-growth rates (see Figure 1).

From the radial velocity of the shell lines of Be shell stars, the relation between $v \sin i$ and the width of the emission lines (e.g. Hanuschik 1996), the peak separation of emission lines arising from outflowing gas (Rivinius et al. 1998, Tarasov \& Roche 1995), and central quasi-emission features in absorption lines of Be stars (Rivinius et al. 1999) it is becoming clear that the disks around Be stars are probably gravitationally supported: the matter orbits in a quasiKeplerian way with radial outflow velocities on the order of that expected for viscous outflow (i.e. $<5 \mathrm{~km} / \mathrm{s}$ ). This could well mean that Waters et al. have overestimated the value of $v_{0}$ by a factor of 4 or so.

As the gas particles in the disk are in (quasi-)Keplerian orbits, their orbit around the star will make them move towards the equatorial plane if they are not already orbiting in that plane. Then the particles moving towards the plane will collide with particles in the plane, leading to new orbits that again will cross the equatorial plane. Consequently, the Keplerian disk can keep itself relatively thin by dissipating the orbits that are tilted to the equatorial plane, while conserving angular momentum. Recent polarimetric observations of the Be star $\zeta$ Tau (Wood et al. 1997) have led to the conclusion that the disk in this Be star has an opening angle of about $\theta \sim 2.5^{\circ}$. This could well mean that Waters et al. have overestimated the value of $\theta$ by a factor of 6 or so. Note that for the model of Waters et al. the disk emission scales with the opening angle if the disk is optically thin: if one assumes a smaller opening angle then the derived density will go up by a factor $\sqrt{\theta / d \theta}$, and this progresses into the derived values of $\dot{M}$. For the optically thick case the densities do not need to 
be rescaled. At the wavelength of the IRAS observations, 12,25 and $60 \mu \mathrm{m}$, the inner part of the disk is thought to be optically thick.

From the above it is clear that if Waters et al. would have used the values of $v_{0}$ and $\theta$ as we currently infer them, their derived mass-loss rates would agree perfectly with the disk-growth rates of X Per, $\mu$ Cen and Pleione.

Note that the above correction on the disk mass-loss rates means that $\mathrm{Be}$ stars lose about $0.5-2 \%$ of their mass on the main-sequence if the stars feed a disk for their entire main-sequence life time.

\subsection{Disk growth: discussion}

I have reviewed evidence that the base densities and growth rate of disks around Be stars depend on the spectral type of the star. The disk mass-loss rates as derived from IR excesses could be made consistent with the observed disk-growth rates. Here I discuss what the consequences are for some of the proposed stellar mass-loss mechanisms that may contribute to the growth of the disks.

The wind compressed disk model: The wind compressed disk model (WCD, Bjorkman \& Cassinelli 1993) presents a mechanism that can funnel a large fraction of the ordinary stellar mass loss to the equatorial plane into the disk. In the bottom panel of Figure 1 the stellar mass-loss rates of luminosity class $\mathrm{V}$ stars are plotted as given by Kudritzki et al. (1989) and Bjorkman \& Cassinelli (1993). These mass-loss rates can directly be compared with the observed diskgrowth rates (and/or with the corrected IRAS disk mass-loss rates). It becomes clear from Figure 1 that if the WCD funnel is $100 \%$ efficient, only the stars with the earliest spectral types have enough "regular" stellar mass-loss to contribute significantly to the observed disk growth. For spectral types B2-B9.5 the WCD mechanism can not contribute significantly to the disk growth, as the stellar mass-loss rates are insufficient (provided that the mass-loss rates of Kudritzki et al. are applicable).

The outburst model: $\quad$ Hipparcos and Hvar photometric surveys (Hubert \& Floquet 1998, Pavlovski et al. 1997) have led to interesting results regarding photospheric outbursts in Be stars. Although these surveys cannot distinguish between magnetic or pulsational origin of the outbursts, they both conclude that short term variations in the form of outbursts are a lot more abundant in earlytype than in late-type Be stars. This may be a clue as to why early-type Be stars have denser disks than late-type Be stars.

In the case of $\mu$ Cen it has been demonstrated that photospheric outbursts actually contribute to disk growth (Hanuschik et al. 1993, Rivinius et al. 1998). Rivinius et al. 1998b) argue that for this star the outbursts occur when the non-radial pulsation modes in the star superpose constructively.

Recently, Henrichs et al. (in press, see also these proc.) have detected the magnetic field of the Be star $\beta$ Cep (which has an atypical low rotational velocity for Be stars). Smith et al. (1997b; these proc.) have analysed the flaring behaviour of the Be star $\lambda$ Eri. This flaring may have a magnetic origin. On one occasion they observed a strong emission peak in a localized velocity region within the HeI 6678 line and modelled the emission as due to a local slab of gas hanging above the photosphere. The mass content in the slab on the order 
of $4 \times 10^{-13} \mathrm{M}_{\odot}$, and it is interesting to note that the star would have to exhibit such flaring about 15 times per day in order to match the disk-growth rate of $\mu$ Cen. In fact, the dimpling rate of this star is of this order (Smith et al. 1996); see Smith et al. (1991) for a discussion on the disk evolution of $\lambda$ Eri. However, for $\lambda$ Eri it has not been demonstrated that the matter that is expelled during a flare contributes to disk growth: it is more likely to fall back to the star (Smith 1994).

\section{Long-term $\mathbf{V} / \mathbf{R}$ variations: global one-armed disk oscillations}

For decades the long-term variability of the shape of the emission line profiles has interested many observers. In particular, the cyclic variations of the ratio of the Violet and Red emission peak strengths, the $V / R$ ratio, has played a prominent role. $\mathrm{V} / \mathrm{R}$ cycle times range from less than two to more than ten years, and the same star can show different cycle times in its observational history. Mennickent et al. (1997) present a list of observational facts such as: the cyclic timescale does not depend on $v \sin i$ or spectral type; the intensity of line emission does not correlate with V/R; the whole profile shifts redwards for $\mathrm{V}>\mathrm{R}$ and vice-versa; in $\gamma$ Cas and 59 Cyg the discrete absorption components (DACs) in UV wind lines are stronger when $\mathrm{V}>\mathrm{R}$.

Hanuschik et al. (1995) list a few of the proposed models for long-term V/R variations: global one-armed oscillations (Okazaki 1991/1996/1997, Papaloizou et al. 1992, Savonije \& Heemskerk 1993), alternating spherical/ellipsoidal infall and outflow (cannot be the case: e.g. Telting et al. 1993, Quirrenbach et al. 1997, Wood et al. 1997), and binarity (cannot be the case: e.g. Telting \& Kaper 1994, Okazaki 1997, Negueruela et al. 1998).

The model with the highest potential of describing all aspects of the longterm $V / R$ variations is that of global one-armed disk oscillations (GOADOs). The model of GOADOs can qualitatively explain the observed V/R cycle times, depending on the basic properties of the disk and on what physical forces are accounted for: deviation of a Keplerian potential due to the oblateness of the star, gas pressure in the disk, radiation pressure, viscosity (see Okazaki, these proc.). The rotational sense of the oscillations, prograde or retrograde, also depends on the above input physics. The GOADO model requires that Be-star disks are (quasi-)Keplerian.

Recent observational studies have focused on line-profile fits, the rotational sense of the oscillations, and the onset of $V / R$ variability. New and more data of V/R variations have been presented by Horaguchi et al. (1994, $\gamma$ Cas), Negueruela (1998, BQ Cam/V0332+53), Reig et al. (1997a, LSI+61235/RX J0146.9+6121).

Line-profile analyses: Hummel \& Vrancken (1995) find from a detailed lineprofile analysis that for $\gamma$ Cas the eccentricity of the elliptic particle orbits decreases with increasing distance from the star, which poses limits on the shape and radial confinement of the disk oscillation. Hanuschik et al. (1995) model Fe II emission line profiles of $66 \mathrm{Oph}$ and $\delta$ Cen as a function of V/R phase, with a GOADO density and velocity perturbation superposed on a disk with a $r^{-2}$ density law. Hummel \& Hanuschik (1997) use a 3D radiative transfer 
code to show that a GOADO density and velocity perturbation in the disk can reproduce all different kinds of symmetric and asymmetric emission line profiles that are observed for Be stars. From these modelling papers it becomes clear that to reproduce the redward profile shift for $V>R$, and vice-versa, one radial node must be present in the GOADO eigenfunction.

Prograde or retrograde?: From the order of the sequence of $\mathrm{V}>\mathrm{R}, \mathrm{V}=\mathrm{R}, \mathrm{V}<\mathrm{R}$, and $\mathrm{V}=\mathrm{R}$ phases, and the relative central shell absorption strength of the profile when $\mathrm{V}=\mathrm{R}$, one can derive whether the disk oscillation revolves progradely or retrogradely (Hummel \& Hanuschik 1994, Telting et al. 1994). Mennickent et al. (1997) give similar arguments to derive the rotational sense of the disk oscillation from photometric measurements. Excellent progress in the observational technique of high-spectral-resolution spatial interferometry has resulted in much more direct ways of determining the rotational sense of the GOADO (Vakili et al. 1998, Berio et al. 1999, see also Stee in these proc.).

So far only prograde GOADOs have been observed in $\beta^{1}$ Mon, $\gamma$ Cas, $\zeta$ Tau. The photometric evidence points to prograde modes in four other Be stars. No retrograde modes have been found to date.

Onset of long-term $V / R$ variability: The observational evidence for the cause of the onset of long-term $V / R$ variations, and hence the triggering of a GOADO, is far from conclusive. Nevertheless there are a few observations which might give useful insight.

Hanuschik et al. (1995) find that in 66 Oph the sudden onset of the V/R variations came after two decades of gradual increase of the emission line strength. By comparison, the onset of the current phase of $\mathrm{V} / \mathrm{R}$ variations in $\gamma$ Cas came after decades of gradual visual brightening, i.e. a gradual increase of the disk emission. Clark et al. (1998a) report the onset of $V / R$ variations, with an exceptionally short cycle time of about a year, in the Be/X-ray binary V725 Tau/A0535+26. Although the observations are sparse also this onset seems to follow a phase of high emissivity. These observations may suggest that the GOADOs are excited when the disk reaches a critical size or density.

Hummel \& Vrancken (1995) find that in Pleione the onset of V/R variability starts in the inner parts of the disk close to the star.

\section{Spectacular variations}

Be stars are known to change from Be to Be-shell and/or diskless phases. Two examples of Be stars that show shell events are $\gamma$ Cas and 59 Cyg. Both stars have shown successive shell events that were accompanied by drastic quasi-cyclic variability of the width of the emission lines, which are called "spectacular variations" (e.g. Doazan et al. 1983). In $\gamma$ Cas the two successive events were followed by an optical low phase in which the star showed only little evidence for the presence of a disk. See Hummel (1998, and references therein) for a detailed description of spectacular variations.

Among proposed models are (see Hummel 1998): successive thick shells, stellar variations, and binarity. Each of these models can only account for some of the aspects of the variations seen during shell events. 
Marlborough (1997) reminds us that the brightness changes during the shell events resemble those of LBV. Perhaps constructive superposition of pulsations may lead to super $\mu$ Cen-like equatorial luminous blue variable-like eruptions?

Recently, Hummel (1998) has proposed a model that can account for the kinematic and geometrical aspects of spectacular variations as seen in the highly variable emission lines: a temporarily tilted disk. Because the disks comes out of the equatorial plane, the variations of the emission-line width are naturally explained if the tilted disk precesses. Theoretical support for possible disk tilt is given by Porter (1998).

The nature of these shell events and associated spectacular variations remains unclear. Modern photometric and spectroscopic data of a new shell event needs to be collected in order to constrain the proposed models.

\section{Disks in $\mathrm{Be} / \mathrm{X}$-ray binaries}

$\mathrm{Be} / \mathrm{X}$-ray binaries are the result of binary evolution. The Roche-lobe overflow and mass transfer stage before the supernova can explain the high rotation rates of Be stars (see Gies, these proc.). A review of $\mathrm{Be} / \mathrm{X}$-ray binaries is given by Coe in these proceedings (see also Negueruela \& Okazaki, these proc.). Here I will concisely discuss the similarity and differences of disk variability in 'single' $\mathrm{Be}$ stars and $\mathrm{Be}$ stars in $\mathrm{Be} / \mathrm{X}$-ray binaries.

Evidence for the hypothesis that disk formation is the same for Be-stars with and without a compact companion comes from the fact that the diskgrowth rates of X Per and $\mu$ Cen are very similar (Hanuschik et al. 1993, Telting et al. 1998). The fact that in $\mathrm{Be} / \mathrm{X}$-ray binaries the $\mathrm{V} / \mathrm{R}$ timescale does not correlate with orbital period (Negueruela et al. 1998) supports the idea that GOADO and V/R timescales are a function of the properties of the Be star and its disk.

Evidence for a compact companion affecting the disks is that in both V725 Tau/A0535+26 and V635 Cas/4U0115+63, giant X-ray outbursts affect the disk: a change of period and phase of $V / R$, and the onset of $V / R$ variations, respectively, have been observed (Clark et al. 1998a, Negueruela et al. 1998). The limiting $\mathrm{H} \alpha$ emission strength is a function of orbital period (Reig et al. 1997b), suggesting that the disk is being trimmed to a certain radius by the companion. The observation that the $\mathrm{V} / \mathrm{R}$ quasi-period is typically shorter in $\mathrm{Be} / \mathrm{X}$-ray binaries (Clark et al. 1998a, Negueruela et al. 1998) supports this idea.

Disks in $\mathrm{Be} / \mathrm{X}$-ray binaries may have a steeper radial density profile than those of "single" Be stars (SS2883/PSR B1259-63, Johnston et al. 1996; X Per, Telting et al. 1998; A0535+26, Clark priv. comm.). This observation is supported by theoretical calculations of disks undergoing close, penetrating encounters with a companion (Hall 1997), although this theory applies to accretion disks.

\section{Conclusions}

The long-term variability in Be stars, and hence the Be phenomenon, still poses many unanswered questions, such as: which stellar mass-loss mechanism is re- 
sponsible for disk growth? I have given evidence that for the WCD model it is difficult to explain the disk-growth rate of the late-type Be star Pleione. Other arguments against the WCD model have been raised by Owocki et al. (1996) and Porter (1997). Observations of $\mu$ Cen show that for this star disk growth is due to pulsationally induced photospheric outbursts. New similar observations of Be stars of different spectral types could prove that this mechanism works for all Be stars.

In my view, the question regarding the origin of long-term cyclic $V / R$ variations has been solved: disk oscillations can account for the observations, but what triggers the excitation of the oscillations is still unclear. The question now is whether we can use a seismological approach to learn about the disk structure and disk growth by observing these oscillations.

The origin of the successive shell events and spectacular variations in $\gamma$ Cas and $59 \mathrm{Cyg}$, which define part of the Be phenomenon, remains unclear. Hopefully $\gamma$ Cas will go through another such event at the end of its current phase of V/R variations, such that we can build up a database of accurate observations to test the models with.

From a comparison of disk variability in $\mathrm{Be} / \mathrm{X}$-ray binaries and normal $\mathrm{Be}$ stars, one can conclude that there is no reason to believe that the stellar massloss mechanism that leads to disk growth is affected by the compact companion. One can also conclude that the timescale of GOADOs and V/R variations are a function of the properties of the Be star and its disk. There is a lot of observational evidence that in $\mathrm{Be} / \mathrm{X}$-ray binaries the structure and size of the disk is affected by the companion star.

Acknowledgments. I thank Myron Smith for his comments on the manuscript.

\section{References}

Balona L.A., Aerts C., Stefl S. 1999, MNRAS, 305, 519

Berio P., Stee Ph., Vakili F., et al. 1999, A\&A, 345, 203

Bjorkman J.E., Cassinelli J.P. 1993, ApJ, 409, 429

Brown A.G.A., Verschueren W. 1997, A\&A, 319, 811

Clark J.S., Tarasov A.E., Steele I.A., et al. 1998a, MNRAS, 294, 165

Clark J.S., Steele I.A., Coe M.J., et al. 1998b, MNRAS, 297, 657

Clark J.S., Lyuty V.M., Zaitseva G.V., et al. 1999, MNRAS, 302, 167

Doazan V., Franco M., Rusconi L., et al. 1983, A\&A, 128, 171

Gehrz R.D., Hackwell J.A., Jones T.W. 1974, ApJ, 191, 675

Hall S.M. 1997, MNRAS, 287, 148

Hanuschik R.W., Dachs J., Baudzus M., Thimm G. 1993, A\&A, 274, 356

Hanuschik R.W., Hummel W., Dietle O., Sutorius E. 1995, A\&A, 300, 163

Hanuschik R.W. 1996, A\&A, 308, 170

Hanuschik R.W., Vrancken M. 1996, A\&A, 312, L17

Hao J.-X., Huang L., Guo Z.H. 1996, A\&A, 308, 499

Harmanec P. 1998, A\&A, 334, 558 
Hirata R. 1995, PASJ, 47, 195

Horaguchi T., Kogure T., Hirata R., et al. 1994, PASJ, 46, 9

Hubert A. M., Floquet M. 1998 A\&A, 335, 565

Hummel W., Hanuschik R.W. 1994, in Proc. IAU Symp. 162 'Pulsation, rotation and mass loss in early type stars', eds. L. Balona, H.F. Henrichs and J.-M.

LeContel, Kluwer, p. 382

Hummel W., Vrancken M. 1995, A\&A, 302, 751

Hummel W., Hanuschik R.W. 1997, A\&A, 320, 852

Hummel W. 1998, A\&A, 330, 243

Johnston S., Manchester R.N., Lyne A.G., et al. 1996, MNRAS, 279, 1026

Juza K., Harmanec P., Bozic H., et al. 1994, A\&AS, 107, 403

Kastner J.H, Mazzali P.A. 1989, A\&A, 210, 295

Koubsky P., Harmanec P., Kubat J., et al. 1997, A\&A, 328, 551

Kudritzki R.P., Pauldrach A., Puls J., Abbott D.C. 1989, A\&A, 219, 205

Kunjaya C., Hirata R. 1995, PASJ, 47, 589

Lyubimkov L.S., Rostopchin S.I., Roche P., Tarasov A.E. 1997, MNRAS, 286, 549

Marlborough J.M. 1997, A\&A, 317, L17

Mennickent R.E., Sterken C., Vogt N. 1997, A\&A, 326, 1167

Mennickent R.E., Sterken C., Vogt N. 1998, A\&A, 330, 631

Moujtahid A., Zorec J., Hubert A.M., et al. 1998, A\&AS, 129, 289

Negueruela I., Roche P., Buckley D.A.H., et al. 1996, A\&A, 315, 160

Negueruela I., Grove J.E., Coe M.J., et al. 1997, MNRAS, 284, 859

Negueruela I., Reig P., Coe M.J., Fabregat J. 1998, A\&A, 336, 251

Okazaki A.T. 1991, PASJ, 43, 75

Okazaki A.T. 1996, PASJ, 48, 305

Okazaki A.T. 1997, A\&A, 318, 548

Oudmaijer R.D., Drew J.E. 1997, A\&A, 318, 198

Owocki S.P., Cranmer S.R., Gayley K.G. 1996, ApJL, 472, 115

Papaloizou J.C., Savonije G.J., Henrichs H.F. 1992, A\&A, 265, L45

Pavlovski K., Harmanec P., Bozic H., et al. 1997, A\&AS, 125, 75

Peters G.J. 1998, ApJL, 502, 59

Poeckert R., Marlborough J.M. 1978, ApJ, 220, 940

Porter J.M. 1997, A\&A, 324, 597

Porter J.M. 1998, A\&A, 336, 966

Quirrenbach A., Bjorkman K.S., Bjorkman J.E., et al. 1997, ApJ, 479, 477

Reig P., Fabregat J., Coe M.J., et al. 1997a, A\&A, 322, 183

Reig P., Fabregat J., Coe M.J. 1997b, A\&A, 322, 193

Reig P., Stevens J.B., Coe M.J., Fabregat J., et al. 1998, MNRAS, 301, 42

Reynolds A.P., Hilditch R.W., Bell S.A., et al. 1992, MNRAS, 258, 439

Rivinius Th., Baade D., Stefl S., et al. 1998, A\&A, 333, 125 
Rivinius Th., Baade D., Stefl S., et al. 1998b, in Proc. 'A Half Century of Stellar Pulsation Interpretations', eds. P.A. Bradley and J.A. Guzik, ASP Conf. Ser. 135, p. 382

Rivinius Th., Stefl S., Baade D. 1999, A\&A, 348, 831

Roche P., Coe M.J., Fabregat J., et al. 1993, A\&A, 270, 122

Roche P., Larionov V., Tarasov A.E., et al. 1997, A\&A, 322, 139

Savonije G.J., Heemskerk M.H.M. 1993, A\&A, 276, 409

Slettebak, A. 1982, ApJS, 50, 55

Smith M.A., Peters G.J., Grady C.A. 1991, ApJ, 367, 302

Smith M.A. 1994, in Proc. IAU Symp. 162, op. cit., ed. L. Balona et al., p. 241

Smith M.A., Hubeny I., Lanz T., Meylan T. 1994, ApJ, 432, 392

Smith M.A., Plett K., Johns-Krull C.M., et al. 1996, ApJ, 469, 336

Smith M.A., Cohen D.H., Hubeny I., et al. 1997a, ApJ, 481, 467

Smith M.A., Murakami T., Ezuka H., et al. 1997b, ApJ, 481, 479

Smith M.A., Robinson R.D., Hatzes A.P. 1998, ApJ, 507, 945

Smith M.A., Robinson R.D. 1999, ApJ, 517, 866

Smith M.A., Roche P.D. 1999, ApJ, 522, 444

Stefl S., Aerts C., Balona L.A. 1999, MNRAS, 305, 505

Tarasov A.E., Roche P. 1995, MNRAS, 276, L19

Telting J.H., Waters L.B., Persi P., Dunlop S. 1993, A\&A, 270, 355

Telting J.H., Kaper L. 1994, A\&A, 284, 515

Telting J.H., Heemskerk M.H., Henrichs H.F., Savonije G.J. 1994, A\&A, 288, 558

Telting J.H., Waters L.B., Roche P., et al. 1998, MNRAS, 296, 785

Unger S.J., Roche P., Negueruela I., et al. 1998, A\&A, 336, 960

Vakili F., Mourard D., Stee Ph., et al. 1998, A\&A, 335, 261

Waters L.B.F.M. 1986, A\&A, 162, 121

Waters L.B.F.M., Coté J., Lamers H.J.G.L.M. 1987, A\&A, 185, 206

Wood K., Bjorkman K.S., Bjorkman J.E. 1997, ApJ, 477, 926

\section{Discussion}

R. Hanuschik: We should be careful with the term "mass-loss rate." In $\mu$ Cen outbursts lasted typically for a few days only, so the amount of actual mass lifted into orbit is much smaller, typically $10^{-11} \mathrm{M} \odot$. Secondly, even the term "mass loss" may be misleading since once matter is lifted, it will circularize quickly and ultimately rain back onto the star. A more appropriate term may be "growth rate" or "outburst rate".

J. Telting: I have used the value of the "mass transfer rate during outbursts" of $\mu$ Cen given by Hanuschik et al. (1993), and corrected that value using the information on the outburst frequency and duration of this star given by Rivinius et al. (1998) and Hubert \& Floquet (1998). The result is used for Figure 1. I don't think that it is clear what fraction of this transferred mass stays in orbit, and how much falls back on the star. 
Even the term "growth rate" is not unambiguous as the observed growth is the net result of what goes into the disk and what leaks out (falling back to the star or moving away at large distances). If the growth rate seems zero this does not mean that the star is not feeding the disk. It means that the disk leaks as much as it is fed. This ambiguity is especially important for the growth rates measured from optical and IR continuum measurements.

J. Bjorkman: I would like to point out, when comparing the disk filling rates with the WCD model predictions, that the WCD predictions are based on theoretical mass-loss rates. These mass-loss rates were calculated using CAK parameters from Abbott (1982). More modern calculations would include many more lines as well as X-ray ionization effects, both of which make the values calculated by Abbott very uncertain. Consequently I am quite pleased that the WCD model approximately reproduces the disk filling rates of the two early type stars. The only real discrepancy is with the remaining late type star for which the mass-loss rates are especially unreliable.

J. Telting: Yes, it is important to realize that the theoretical mass-loss rates for (rapidly rotating) B stars may need a closer look.

M. Smith: Regarding the one-armed disk around $\gamma$ Cas, the Berio et al. (1999) paper you quote places the disk's 'inner edge' distance from the star for the first time. This disk is at a similar distance to the star as the clouds for this star (Smith, these proc.). Robinson and I believe the X-rays from this star could be created or at least modulated by a star-disk magnetic interaction, so it is possible that the mean X-ray flux will modulate with the disk precession cycle. S. Owocki: Since the global disk oscillations are so successful in explaining the $\mathrm{V} / \mathrm{R}$ variations it is important to remember that a key requirement for these oscillations is that the disks have stable Keplerian orbits. In contrast, the wind compressed disk does not provide angular momentum needed to let material be in orbit, and so it falls back on the star. Instead of the few-years timescale for the $V / R$ changes, material only stays in the disk for a few days. In my eyes, this is one of the strongest pieces of evidence against the WCD model.

J. Telting: I agree. Regarding the travel time of the gas in the disk: for X Per a disk-loss event lasts approximately as long as a complete build-up, i.e. a timescale of about a year. If one can regard the disk as a reservoir through which material is slowly moving outwards, this means it takes about a year for the material to reach the outer radius of the visible disk, which for the optical light curve is at a few stellar radii.

T. Rivinius: About radial outflow velocities, I would like to add that we have estimated this to $\sim 10 \mathrm{~km} / \mathrm{s}$ in $\mu$ Cen from the variation of the peak separation. From the formation mechanism of central quasi emission bumps (see my poster presentation) we derive even lower values.

These CQEs do not appear in stars with $\mathrm{V} / \mathrm{R}$ activity, probably because there are radial velocity fields due to the elliptical orbits in these disks.

J. Telting: I am convinced that radial outflow velocities in Be-star disks are much smaller than previously thought. When you say that the outflow velocity in the disk of $\mu$ Cen is smaller than $\sim 10 \mathrm{~km} / \mathrm{s}$ I assume that this is an average value over the line formation region, and that hence the outflow velocity is low even at considerable distance of the star. This means that there is probably little radial acceleration in the disk. 